\title{
An accident and emergency based child accident surveillance system: is it possible?
}

\author{
T F Beattie
}

\begin{abstract}
Objective-To evaluate the possibility of setting up a database on childhood accidents within an accident and emergency (A\&E) department.

Design-A proforma detailing epidemiological details about the child and details of the injury was designed and tested for accuracy. It was completed in parallel with the existing case documentation. A retrospective sample was analysed after completion of the study to determine times when data collection was poorest. Setting-Regional paediatric hospital (total catchment population c. 500000 ). Results-Of 13958 patients in whom full information was available, $65 \%$ had all the information available on the forms. Areas of discrepancy in the remainder included (1) transposition of date and time of injury with date and time of attendance $(73 \%)$, (2) wrong coding (11\%), (3) illegible digits $(6 \%)$, (4) inaccurate data entry to computer $(6 \%)$. Form completion was worst between 1800 hours and midnight.

Conclusions-Data collection is feasible in the A\&E department and is a necessary step in effective child accident prevention. It should be done using real time entry onto computer systems. Additional audit staff must be employed to ensure data collection is as complete as possible as close to the time of the initial attendance as possible. Regular analysis of the findings is essential.

( $f$ Accid Emerg Med 1996;13:116-118)
\end{abstract}

Key terms: accidents; accident prevention; accident surveillance; accident and emergency department

Childhood accidents account for two million attendances to accident and emergency (A\&E) departments throughout the United Kingdom each year. Between 800 and 900 children will die as a result of these accidents. A further 10000 will be seriously injured or disfigured. Indeed childhood accidents are the leading cause of mortality between the ages of 1 and 14 , being responsible for more deaths than cancer or infectious disease. ${ }^{1}$

Clearly there is a need for action to try and reduce this mortality and morbidity. Any effort to reduce the incidence and severity of childhood accidents however relies on five prime steps: (1) development of a baseline database; (2) from the database identify problem areas; (3) development of strategies potentially effective against the problems identified in (2);
(4) implementation of the strategies; (5) audit by comparison with baseline database.

Once this cycle is established a new database will exist and the cycle can begin again. There is therefore a need to set up an effective database for childhood accidents. This concept is not new, with many other specialties possessing illness and disease surveillance and audit systems. Indeed other countries have taken the lead in developing childhood accident surveillance systems. ${ }^{23}$

Within the United Kingdom several organisations already audit childhood accidents but each of these has its own problems. The Department of Transport and Industry compiles a sample of accidents from around the country and extrapolates national figures from this (Home Accident Surveillance System: $\mathrm{HASS}^{4}$ ). Police gather statistics on road accidents; the fire brigade collect information on house fires and some thermal injuries; the Coroner in England and the Procurator Fiscal in Scotland have details of accidental death; the Scottish Office or the Department of Health in England has details of hospital discharges. If pooled, these would have the potential to measure accidents over a given period of time. However, these results are seldom pooled and each exists on its own, with very little relevance to individual communities.

With regard to HASS, the collection of data is admitted by the compilers to be incomplete and data collection is poor when trained researchers are absent from the departments included in the survey. ${ }^{4}$

It has recently been shown that in the case of road traffic accidents there is a discrepancy between police and hospital figures. ${ }^{5}$ It has also been shown that coding performed in certain circumstances is inaccurate and that statistics derived from discharge codes may need to be re-evaluated. ${ }^{6}$

The one area that sees most of the accidents that happen in childhood is the A\&E department. Little attention has previously been paid to the development of this resource as a source of statistics. Indeed much of the development has been hampered by the lack of computerisation in $\mathrm{A} \& \mathrm{E}$ departments.

This paper examines the development of a child accident database in the $\mathrm{A} \& \mathrm{E}$ department of the Royal Aberdeen Children's Hospital over a one year period. The system was manual but was designed to act mainly as a pilot project to facilitate the subsequent development of a computerised system for accident surveillance.

The purpose of this paper is to evaluate the possibility of setting up a database on 
childhood accidents within the accident and emergency department. No funding was available for computer documentation but in any case before computer documentation can be achieved, some idea as to the feasibility of data collection has to be made.

\section{Methods}

SETTING

The Royal Aberdeen Children's hospital is the sole paediatric hospital in the North East of Scotland. It has a catchment area of approximately 500000 people. However the bulk of the catchment population resides in the Aberdeen area which has a population of 250000 . The paediatric population in this area is 50000 . Children outwith a 10 mile radius are much more likely to seek help from their general practitioner or the local cottage hospital. However, all serious injuries and those occurring outside "office hours" will present to the A\&E department of the Royal Aberdeen Children's Hospital. Many of the rest will be referred for further investigation and treatment at the $A \& E$ department.

The A\&E department sees something in excess of 16000 children each year, which is a $90 \%$ increase from 25 years ago. ${ }^{8}$

DATA COLLECTION

The year long survey was planned to run from 00.01 hours on 18 December 1989 to midnight on 17 December 1990. All children attending the $A \& E$ department between these dates were eligible for admission.

A proforma was designed detailing epidemiological details about the child together with information regarding the nature and causation of the injury. Half the form was expected to be completed for each patient but with other areas being completed only if relevant. The form was A3 in size. It was designed in duplicate using "no carbon required" (NCR) paper. The top copy was attached to the permanent casualty record with the bottom copy going for analysis. The main reason for this was to prevent doctors having to duplicate information.

Every child attending the A\&E department had a casualty card filled out as usual. This was then processed together with a copy of the surveillance form. The surveillance form was completed in part by the receptionist and the clinical details were then entered by the doctor at the same time as they saw the patient. Completed forms were processed as below.

DATA HANDLING

Agreement was reached with the computing department of the Department of Public Health Medicine, Grampian Health Board. A computer program was written in COBOL on an ICL 3900 series mainframe. It was designed to handle all the required data. Completed forms were collected in the A\&E department and forwarded at regular intervals to the data processing department. The forms were checked initially for legibility and then subsequently for accuracy. Data were entered manually onto the computer. Various checks for accuracy were built into the computer program and should any information be deemed inaccurate the forms were rejected and returned to the $A \& E$ department for correction.

Mistakes were classed as either correctable or uncorrectable. Correctable mistakes included: (1) transposition of date and time of injury with date and time of attendance; (2) incorrect date of birth; (3) inaccurate coding; (4) incomplete data. All of these only pertained if the accurate information was present on the $A \& E$ record card. Otherwise details were deemed uncorrectable.

Forms were deemed uncorrectable when: (1) data omitted from the form were not present in the clinical notes; (2) date and time of injury were not documented on the clinical notes; (3) wrong coding, where information could not be correctly gleaned from the inpatient or $\mathrm{A} \& \mathrm{E}$ notes.

A retrospective sample was taken after completion of the study in an effort to identify times when data collection was poorest. Sampling was done on day 1 and each eighth day thereafter. Forms were identified for each of these days and compared with the actual attendances documented in the register. The times at which form completion was poorest was analysed.

\section{Results}

The study took place from 00.01 hours on 18 December 1989 to midnight on 17 December 1990 . During the period of the study a total of 16402 patients were treated at the $A \& E$ department according to the register at the front desk. Further analysis of this revealed that $\mathbf{4 4 3}$ were over age (for example, parents, relatives, staff), return visits with the same problems, or had been doubly documented. This left a total of 15959 children eligible for inclusion in the study.

Useful information was returned on 13968 patients $(87.5 \%)$. Of the remaining 1991 patients no forms were available for 556 patients and 1435 had incomplete data. This latter group had forms raised but the data were incomplete and uncorrectable. With regard to incomplete data, absent date and/or time of injury accounted for $637(44 \cdot 3 \%)$ of the total. Illegible codes, missing records, and incomplete coding accounted for the rest. Of the 1991 patients, $1069(74.5 \%)$ fell into the nonaccident category, for example, illness, atraumatic infection, abdominal pain, and so on.

Of the 13958 patients for whom full information was available, $65 \%$ had all of the information available on the forms. A further $35 \%$ had forms completed with reference to casualty cards after the patient had been discharged. The areas of discrepancy were as follows:

(1) Transposition of date and time of injury with date and time of attendance $(73 \%)$

(2) Wrong coding (11\%)

(3) Illegible digits $(6 \%)$

(4) Inaccurate data entry to computer (6\%)

(5) No abnormality in the form but rejected by computer $(5 \%)$

The main problem with coding concerned the misclassification of children who had been 
poisoned as medical problems. A simple alteration in the computer program corrected this automatically.

With regard to the time of day when the form completion was poorest, the sampling procedure identified 1821 attendances. Of these 1547 had forms completed (84.9\%). Form completion was poorest between 1800 hours and midnight $(72.9 \%)$ compared with $91 \cdot 4 \%$ during the rest of the day.

\section{Discussion}

This project is the first step towards designing a data collection system to enable us to derive a database with regard to childhood accidents. It suffers from several flaws. Firstly, we only collected information on $87.5 \%$ of those eligible for inclusion. This compares favourable with the range of $65-78 \%$ data collection from the Western Australia surveillance study. ${ }^{2}$ It falls short of what I would consider optimal data collection: ideally forms would have been completed for every child who attended and they would have been checked at least daily to ensure accuracy, legibility, and completeness. Missing data could have been sought, either on the child's return visit, by questioning the child in hospital, or by contacting family and relatives directly while information regarding the injury was fresh in their mind. Lack of funding meant that we were unable to employ a specific audit clerk to do this. Data collection was particularly bad for the first fortnight of the study and it was not until remedial efforts were made that collection improved significantly. Data collection was also extremely poor in the last fortnight of the study, probably due to doctor fatigue.

Secondly we suffered significantly from not being able to enter data in real time. Data were collected contemporaneously but were not entered into the computer for several weeks after they were collected. It was by this stage too late to correct wrong data or to gather absent data. This is not to criticise data processors and data processing staff. Lack of funding meant we were unable to pay for dedicated data processing. Use was made of the facilities available within Grampian Health Board and data were entered when time became available and when no other pressing needs existed.

Apart from the periods at the beginning and end of the study, no significant variation in data collection existed that could be accounted for by seasonal or temporal factors. With regard to time of data collection, collection was very much poorer during the evening than during the rest of the day, with only $73 \%$ being completed during the hours of 1800 hours to midnight shift. This is usually the busiest time of the day in the department with a single handed doctor seeing large numbers of patients. This mirrors the experience of HASS and Western Australia. ${ }^{24}$

The commonest mistake made on the form was for doctors to transpose the date and time of injury. Given the pressure that doctors were under it is not surprising that this occurred so often.
Analysis of the incompletely documented or absent forms was revealing. Seventy five per cent of these fell into a "non-accidental" category. This would suggest that doctors discriminated positively towards accidents and made every effort possible to fill out forms for accidents. It would appear that this occurred to the exclusion of the non-accident categories.

An early set of results was made available after four months of the study had taken place and were presented to all people involved in data collection. There is no doubt that this greatly increased their awareness of the problems and they welcomed the feedback. Unfortunately it was not possible to do this again until after the study had been completed. Again this is a reflection of the manual nature of the system and the lack of funding for additional staff.

Several lessons have been learned from this exercise. Firstly, data collection is possible in the $A \& E$ department. These data can and will be made available to the relevant health promotion agencies and hopefully effective child accident prevention can begin. Secondly, any further data collection must be made real time using real time entry onto computer systems. Thirdly, additional audit staff must be employed to ensure data collection is as complete as possible and as close as possible to the time of the initial attendance. It is not feasible to continue an audit of this magnitude without employing additional staff who can follow up incomplete data to ensure that data collection and capture is as near $100 \%$ as possible. Fourthly, regular analysis must be made of the findings from any such audit. Staff very much appreciated the feedback given but said that they would probably have been more encouraged were this to be presented weekly rather than annually.

In conclusion, this one-off exercise has proven the worth of the $A \& E$ department as a reasonable focus for data collection for a child accident surveillance system. However, further work needs to be done to improve data collection techniques.

My thanks are due to Miss Rebecca Moir and Mrs Elaine Lord for preparing the manuscript; to the staff of the Information Technology Centre, Grampian Health Board; Mr E Alexander for writing a comprehensive computer program and putting up for writing a comprehensive computer program and putting up
with my foibles; to $\mathrm{Mr}$ G Bell for designing the proforma used in the study; to Mrs Lesley Tock for card and record retrieval. This study was made possible by donation of a prize won by making this prize available to me for this work.

1 Basic principles of child accident prevention: a guide to action. London: Child Accident Prevention Trust, 1989.

2 Annual Report of the Western Australian Childhood Injury Surveillance System. Child Accident Prevention Foundation of Australia, (West Australia Division), 15 Hamilton St, Subiaco 6008, W Austrailia, 1989.

3 Peclet M, Newman K, Eichelberger MR, Gotschall CS, Guzetta PC, Anderson KD, et al. Patterns of injury in Guzetta PC, Anderson KD, et al. Pate

4 Home and leisure accident research; 11 th annual report. London: Department of Trade and Industry, 1989.

Teanby $D$. Underreporting of pedestrian road accidents. BMF 1992;304:422

6 Muchison J, Barton J, Ferguson A. An analysis of cases incorrectly coded as inflammatory bowel disease in incorrectly coded as inflammatory bowel disease in Scottish Hospital

7 Jones PF, Karmody A M, Galloway JMD. A children's casualty department. BMF 1966;ii:819-21. 